\title{
Genetically Engineered Plasmonic Nano-Arrays
}

\author{
Carlo Forestiere,${ }^{\dagger, \dagger}$ Alyssa J. Pasquale, ${ }^{\dagger}$ Antonio Capretti, ${ }^{\ddagger \dagger}{ }^{\dagger}$ Giovanni Miano, ${ }^{\ddagger}$
} Antonello Tamburrino, "I Sylvanus Y. Lee, ${ }^{\dagger, \S}$ Björn M. Reinhard, " and Luca Dal

$$
\text { Negro*, } \dagger
$$

Department of Electrical and Computer Engineering and Photonics Center, Boston University, 8

Saint Mary's Street, Boston, Massachusetts, 02215, Department of Electrical Engineering,

Università degli Studi di Napoli Federico II, via Claudio 21, Napoli, 80125, Italy, DAIEMI, Università degli Studi di Cassino, Via G. Di Biasio 43, Cassino, 03043, Italy, Department of Mechanical Engineering, Boston University, 110 Cummington St., Boston, Massachusetts 02215, and Department of Chemistry, Boston University, 590 Commonwealth Avenue, Boston,

Massachusetts, 02215

E-mail: dalnegro@bu.edu

\section{Supporting Information Available}

Generalized Multiparticle Mie Theory. This section introduces the basic concepts of the Generalized Multiparticle Mie (GMM) theory and it is mainly based on the works of Xu. ${ }^{1,2}$ We refer to these works and to the references therein for further details.

\footnotetext{
${ }^{*}$ To whom correspondence should be addressed

†Department of Electrical and Computer Engineering and Photonics Center, Boston University, 8 Saint Mary's Street, Boston, Massachusetts, 02215

*Department of Electrical Engineering, Università degli Studi di Napoli Federico II, via Claudio 21, Napoli, 80125, Italy

IIDAIEMI, Università degli Studi di Cassino,Via G. Di Biasio 43, Cassino, 03043, Italy

${ }^{\S}$ Department of Mechanical Engineering, Boston University, 110 Cummington St., Boston, Massachusetts 02215

"Department of Chemistry, Boston University, 590 Commonwealth Avenue, Boston, Massachusetts, 02215
} 
Let us consider a set of $L$ non-overlapping spherical particles, shown in Figure 1, whose $j^{\text {th }}$ element has radius $a^{j}$ and is centered at $\left(x^{j}, y^{j}, z^{j}\right)$ in the primary reference system $(0, \mathrm{X}, \mathrm{Y}, \mathrm{Z})$. We introduce a number $L$ of local reference systems $\left(0, \mathrm{X}^{\mathrm{j}}, \mathrm{Y}^{\mathrm{j}}, \mathrm{Z}^{\mathrm{j}}\right)$ with $j=1 \ldots L$, centered on the $j^{\text {th }}$ particle and with parallel axes to those of the primary system. The field scattered by the $j^{t h}$ particle

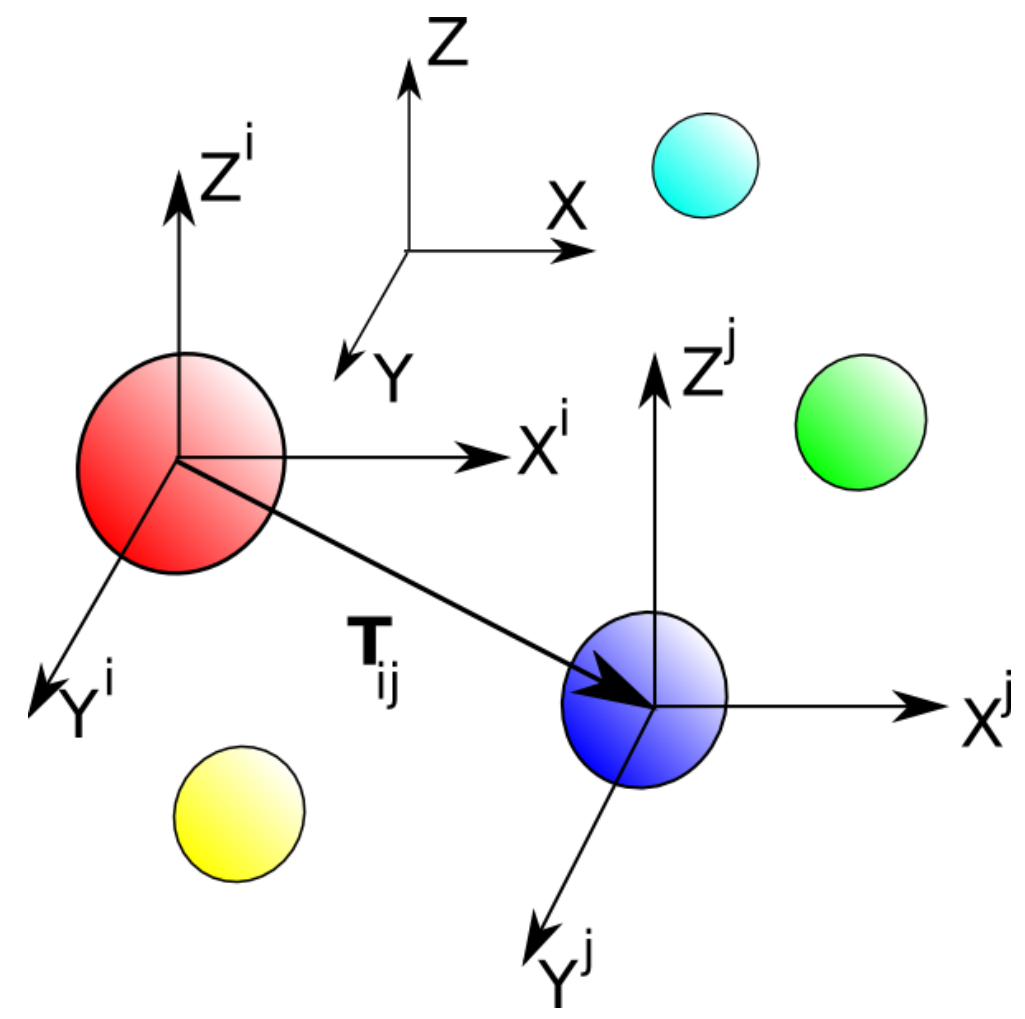

Figure 1: Geometry of the scattering problem.

can be expressed in terms of the radiating Vector Spherical Wave Functions (VSWFs) set centered on the $j^{\text {th }}$ particle itself, that is

$$
\left\{\begin{array}{l}
\mathbf{E}_{s}^{j j}=\sum_{n=1}^{\infty} \sum_{m=-n}^{n} j E_{m n}\left[a_{m n}^{j j} \mathbf{N}_{m n}^{3}\left(k_{0} r^{j}, \theta^{j}, \phi^{j}\right)+b_{m n}^{j j} \mathbf{M}_{m n}^{3}\left(k_{0} r^{j}, \theta^{j}, \phi^{j}\right)\right] \\
\mathbf{H}_{s}^{j j}=\frac{k_{0}}{\omega \mu_{0}} \sum_{n=1}^{\infty} \sum_{m=-n}^{n} E_{m n}\left[b_{m n}^{j j} \mathbf{N}_{m n}^{3}\left(k_{0} r^{j}, \theta^{j}, \phi^{j}\right)+a_{m n}^{j j} \mathbf{M}_{m n}^{3}\left(k_{0} r^{j}, \theta^{j}, \phi^{j}\right)\right]
\end{array}\right.
$$

The same field scattered by the $j^{\text {th }}$ particle, can be also expanded into the VSWF set centered on 
the $i^{\text {th }}$ particle, as follows:

$$
\left\{\begin{array}{l}
\mathbf{E}_{s}^{j i}=\sum_{n=1}^{\infty} \sum_{m=-n}^{n} j E_{m n}\left[a_{m n}^{j i} \mathbf{N}_{m n}^{3}\left(k_{0} r^{i}, \theta^{i}, \phi^{i}\right)+b_{m n}^{j i} \mathbf{M}_{m n}^{3}\left(k_{0} r^{i}, \theta^{i}, \phi^{i}\right)\right] \\
\mathbf{H}_{s}^{j i}=\frac{k_{0}}{\omega \mu_{0}} \sum_{n=1}^{\infty} \sum_{m=-n}^{n} E_{m n}\left[b_{m n}^{j i} \mathbf{N}_{m n}^{3}\left(k_{0} r^{i}, \theta^{i}, \phi^{i}\right)+a_{m n}^{j i} \mathbf{M}_{m n}^{3}\left(k_{0} r^{i}, \theta^{i}, \phi^{i}\right)\right]
\end{array}\right.
$$

The vector addition theorem for VSWF enables the transformation of the series expansion coefficients $a_{m n}^{j j}$ and $b_{m n}^{j j}$ for the scattered fields of the $j^{t h}$ particle into an expansion in the local coordinate system associated with the $i^{t h}$ particle $a_{m n}^{j i}$ and $b_{m n}^{j i}$, as follows:

$$
\left\{\begin{array}{l}
a_{m n}^{j i}=\sum_{v=1}^{\infty} \sum_{\mu=-v}^{v} A_{m n, \mu v}^{j i} a_{\mu \nu}^{j j}+B_{m n, \mu v}^{j i} b_{\mu v}^{j j} \\
b_{m n}^{j i}=\sum_{v=1}^{\infty} \sum_{\mu=-v}^{v} B_{m n, \mu v}^{j i} a_{\mu v}^{j j}+A_{m n, \mu v}^{j i} b_{\mu \nu}^{j j}
\end{array}\right.
$$

The translation matrices $A_{m n, \mu v}^{j i}$ and $B_{m n, \mu v}^{j i}$ only depend on the distance and direction of the translation vector from the center of the $i^{t h}$ NP to the center of the $j^{\text {th }}$ NP. The derivation of the translational matrices can be greatly simplified by decomposing the overall translation into a combination of two rotations and a translation along the z-axis, according to the Mackowski three-step procedure. $^{2,3}$

The external field incident on the $i^{\text {th }}$ sphere can be expanded into regular VSWFs associated to the reference system $\left(0^{i}, X^{i}, Y^{i}, Z^{i}\right)$

$$
\left\{\begin{array}{l}
\mathbf{E}_{e}^{i i}=-\sum_{n=1}^{\infty} \sum_{m=-n}^{n} j E_{m n}\left[p_{m n}^{i i} \mathbf{N}_{m n}^{1}\left(k_{0} r^{i}, \theta^{i}, \phi^{i}\right)+q_{m n}^{i i} \mathbf{M}_{m n}^{1}\left(k_{0} r^{i}, \theta^{i}, \phi^{i}\right)\right] \\
\mathbf{H}_{e}^{i i}=-\frac{k_{0}}{\omega \mu_{0}} \sum_{n=1}^{\infty} \sum_{m=-n}^{n} E_{m n}\left[q_{m n}^{i i} \mathbf{N}_{m n}^{1}\left(k_{0} r^{i}, \theta^{i}, \phi^{i}\right)+p_{m n}^{i i} \mathbf{M}_{m n}^{1}\left(k_{0} r^{i}, \theta^{i}, \phi^{i}\right)\right]
\end{array}\right.
$$

In the reference system $\left(0^{i}, \mathrm{X}^{\mathrm{i}}, \mathrm{Y}^{\mathrm{i}}, \mathrm{Z}^{\mathrm{i}}\right)$ the electric field on the $i^{\text {th }}$ sphere due to all other particles and the external field, is:

$$
\mathbf{E}_{i}^{i i}=\sum_{\substack{j=1 \\ j \neq i}}^{L} \mathbf{E}_{s}^{j i}+\mathbf{E}_{e}^{i i}
$$


Therefore, since the problem has been reduced to single scattering we can now utilize the single particle Mie theory ${ }^{1}$ to calculate the overall scattering coefficients of the $i^{\text {th }}$ sphere:

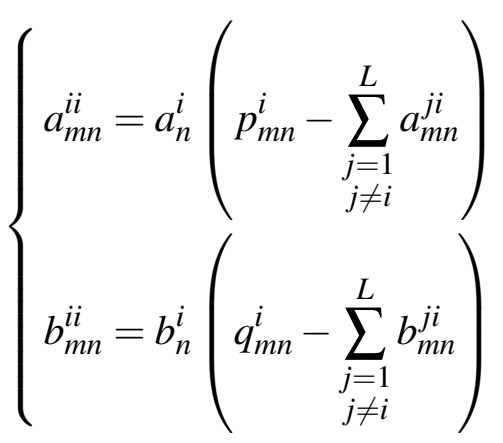

where $i \in\{1,2, \ldots L\}, n \in\{1,2, \ldots, \infty\},|m| \leq n$, while $a_{n}^{i}$ and $b_{n}^{i}$ are the single sphere Mie theory coefficients. ${ }^{4}$ By using the addition theorem, i.e. eqs. 3 , into eqs. 6 we have:

$$
\left\{\begin{array}{c}
a_{m n}^{i i}+a_{n}^{i} \sum_{\substack{j=1 \\
j \neq i}}^{L} \sum_{v=1}^{\infty} \sum_{\mu=-v}^{v}\left[A_{m n, \mu \nu}^{j i} a_{\mu \nu}^{j j}+B_{m n, \mu \nu}^{j i} b_{\mu \nu}^{j j}\right]=a_{n}^{i} p_{m n}^{i} \\
b_{m n}^{i i}+b_{n}^{i} \sum_{\substack{j=1 \\
j \neq i}}^{L} \sum_{v=1}^{\infty} \sum_{\mu=-v}^{v}\left[B_{m n, \mu v}^{j i} a_{\mu v}^{j j}+A_{m n, \mu v}^{j i} b_{\mu v}^{j j}\right]=b_{n}^{i} q_{m n}^{i}
\end{array}\right.
$$

where $i \in\{1,2, \ldots L\}, n \in\{1,2, \ldots, \infty\}$, and $|m| \leq n$. In order to numerically solve the system of eqs. 7 we truncate the scattered field and the incident field expansion of the particles to a finite $\operatorname{order} N_{\max }$, namely:

$$
\left\{\begin{array}{c}
a_{m n}^{i i}+a_{n}^{i} \sum_{\substack{j=1 \\
j \neq i}}^{L} \sum_{v=1}^{N_{m a x}} \sum_{\mu=-v}^{v}\left[A_{m n, \mu v}^{j i} a_{\mu \nu}^{j j}+B_{m n, \mu v}^{j i} b_{\mu \nu}^{j j}\right]=a_{n}^{i} p_{m n}^{i} \\
b_{m n}^{i i}+b_{n}^{i} \sum_{\substack{j=1 \\
j \neq i}}^{L} \sum_{v=1}^{N_{m a x}} \sum_{\mu=-v}^{v}\left[B_{m n, \mu v}^{j i} a_{\mu \nu}^{j j}+A_{m n, \mu \nu}^{j i} b_{\mu \nu}^{j j}\right]=b_{n}^{i} q_{m n}^{i}
\end{array}\right.
$$

where $i \in\{1,2, \ldots L\}, n \in\left\{1,2, \ldots, N_{\max }\right\}$, and $|m| \leq n$. The system of eqs. 8 was solved iteratively using the approach proposed by $\mathrm{Xu}{ }^{1}$

Genetic Algorithm. In the framework of GAs, ${ }^{5}$ a feasible solution of the given optimization problem, usually referred to as an individual, is represented by a binary string, namely a chro- 
mosome. Each chromosome is in turn subdivided into $N$ genes; the $j^{\text {th }}$ gene represents, through Gray coding, the $j^{\text {th }}$ degree of freedom of the problem. The choice of Gray coding guarantees that two consecutive values of a given degree of freedom are represented by binary sequences that differ only in one digit. Each chromosome is given a fitness value. The implemented GA works as follows: i) the population, which consists of $W$ individuals, is randomly initialized. ii) The fitness of each individual is evaluated and the selection of the mating pool among the population is performed. The implemented selection criterion is the roulette wheel selection: each wheel's slot is proportional to the individual fitness. Indeed, a fit individual has a better probability of being selected for mating. iii) Once the mating has been performed the crossover process between two chromosomes can take place. The two mating chromosomes are cut at the same randomly selected point and the resulting strings are swapped between them. iv) In order to prevent the GA from becoming trapped in local minima, and to preserve the genetic diversity of the population, the chromosomes are subjected to mutation. The implemented mutation consists of inverting the value of one bit along the chromosome with a given probability $P_{m}$. v) Eventually the old generation is killed, replaced by new individuals, providing that the best individual in the old population is maintained (elitism). vi) Once the iteration number reaches the maximum value $N_{\text {iter }}$ the GA stops, otherwise the iterative process restarts from step ii. In all the GA optimizations performed in this paper we have assumed a population $W$ of 48 individuals, a number of iterations $N_{\text {iter }}=10000$, and a probability of mutation of $P_{m}=0.1$.

Two Dimensional Optimization with Varying NPs Size. This analysis consists in the synthesis of both the NPs centers and radii, assuming $\delta=3 \mathrm{~nm}, R_{\min }=5 \mathrm{~nm}, R_{\max }=150 \mathrm{~nm}$, and an incident wavelength $\lambda^{(A g)}=370 \mathrm{~nm}$ for $\mathrm{Ag}$ and $\lambda^{(A u)}=535 \mathrm{~nm}$ for Au. The optimized patterns follow the same physical rules found on the basis of the analysis discussed in the text, even if the additional degree of freedom leads to better performances.

We found that regardless of the metal choice the $N=2$ configuration of NPs results in an asymmetric dimer, i.e. composed of particles with different radii, aligned along the polarization direction, with the minimum allowed edge-to-edge spacing. We show this configuration in Figure 2 
(a) for $\mathrm{Au}$ and (e) for $\mathrm{Ag}$, while the exact particle coordinates are listed in Table 1. When more particles are added to the system, for instance when $N=6$ as shown in Figure 2 (b), we find that $\mathrm{Au}$ NPs assemble to form asymmetric dimers, placed symmetrically with respect to the probing point along the vertical direction in order to provide an in-phase radiative contribution. In the $\mathrm{Ag}$ NP array with $N=6$, shown in Figure 2 (f), the near field resonance supported by the central dimer is boosted by the far field radiative coupling of the remaining particles. The radius of the particles in the far zone turns out to be the maximum allowed $\left(R_{\max }=150 \mathrm{~nm}\right)$, in this way the radiative contribution is maximized and the achieved field enhancement is twice what is found in the corresponding fixed radius scenario.

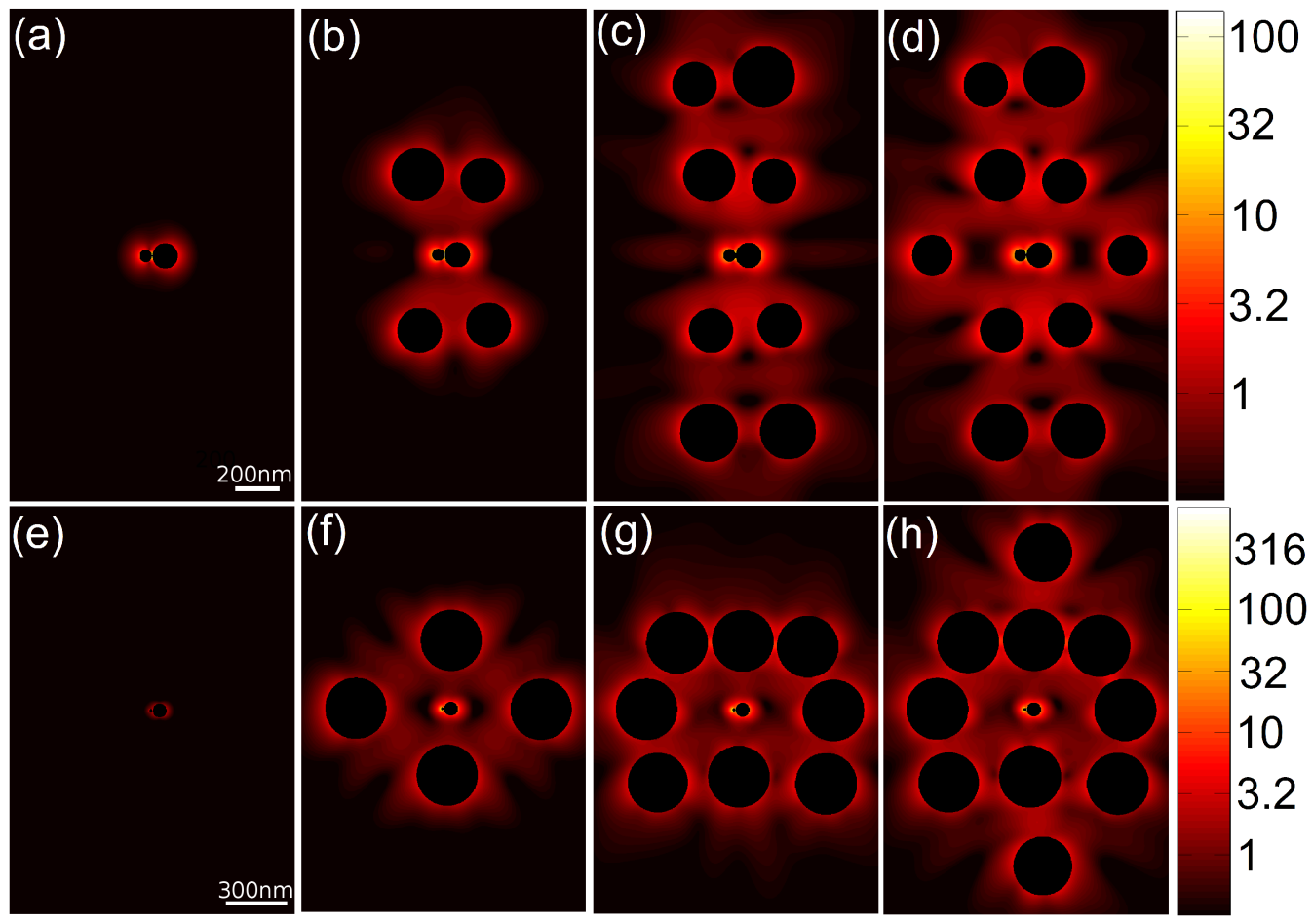

Figure 2: Electric field magnitude on the equatorial plane of the optimized NP arrays composed of (a,e) $\mathrm{N}=2,(\mathrm{~b}, \mathrm{f}) 6,(\mathrm{c}, \mathrm{g}) 10$ and $(\mathrm{d}, \mathrm{h}) 12 \mathrm{Au}$ and Ag spheres, respectively. The achieved values of field enhancement in $\mathbf{r}_{0}\left(\mathbf{r}_{0}\right.$ is the center of each simulation box) are (a) 64 (b) 121 (c) 154 (d) 165 for $\mathrm{Au}$ and (e) 218, (f) 557, (g) 676 (h) 760 for Ag. The color maps are in logscale.

When increasing the NP number to $N=10$ we find that, in the Au case, a second order grating 
contribution is required to further enhance the field as shown in Figure 2 (c). In Ag case, shown in Figure $2(\mathrm{~g})$, the central dimer is surrounded by a NP necklace ${ }^{6}$ responsible for the radiative boosting, which leads to a FE increase of more than $20 \%$ with respect of the $\mathrm{N}=6$ case. Finally, by adding further NPs, we found that also for Ag arrays the last two particles are placed further away to maximize the near-field at the probing point via in phase second order radiative contribution. However, we can notice that, as in the two dimensional optimization with fixed radius, the fundamental NP units turn out to be the isolated particle in the case of Ag aggregates and NP dimers for $\mathrm{Au}$ patterns, with the difference that they are asymmetric.

Table 1: Optimized 2D arrays of Au and Ag NPs.

\begin{tabular}{r|rrrc|rrrr}
\hline & \multicolumn{4}{|c}{$\mathrm{Au}$} & \multicolumn{5}{c}{$\mathrm{Ag}$} \\
\hline \hline $\mathrm{N}$ & $\mathrm{x}[\mathrm{nm}]$ & $\mathrm{y}[\mathrm{nm}]$ & $\mathrm{R}[\mathrm{nm}]$ & $\mathrm{FE}_{0}$ & $\mathrm{x}[\mathrm{nm}]$ & $\mathrm{y}[\mathrm{nm}]$ & $\mathrm{R}[\mathrm{nm}]$ & $\mathrm{FE}_{0}$ \\
\hline 1 & 64 & 0 & 61 & & 36 & 0 & 33 & \\
2 & -29 & 0 & 29 & 63 & -6.5 & 0 & 6.5 & 218 \\
\hline 3 & 215 & -340 & 108 & & 480 & 0 & 150 & \\
4 & -119 & -364 & 108 & & -433 & 0 & 150 & \\
5 & -128 & 390 & 127 & & 17.6 & -328 & 150 & \\
6 & 187 & 362 & 108 & 121 & 37.2 & 337 & 150 & 557 \\
\hline 7 & 138 & 871 & 150 & & 354 & 309 & 150 & \\
8 & 501 & 0 & 99 & & -284 & 327 & 150 & \\
9 & 256 & -860 & 135 & & -392 & -362 & 150 & \\
10 & -129 & -865 & 140 & 154 & 378 & -355 & 143 & 676 \\
\hline 11 & -463 & 0 & 96 & & 156 & 776 & 106 & \\
12 & -200 & 833 & 108 & 165 & 156 & -776 & 106 & 760 \\
\hline
\end{tabular}

FDTD Analysis Details All particles are modeled using material dispersion data from Johnson and Christy, ${ }^{7}$ using particle shapes and sizes based on SEM of the fabricated particles. Meshing in the $\mathrm{x}$ and $\mathrm{y}$ (in-plane) dimensions is $2.5 \mathrm{~nm}$ around all nanoparticles, and $2 \mathrm{~nm}$ in the $\mathrm{z}$ dimension (out-of-plane) in order to ensure numerical convergence. All arrays are simulated on a glass substrate in order to exactly describe our experimental conditions. In all simulations, plane wave excitation was used at normal incidence to the substrate. All periodic structures were simulated with periodic boundary conditions, otherwise perfectly matched layer (PML) boundary conditions were used to absorb all electromagnetic radiation at the simulation boundaries. ${ }^{8}$ In the case of geometries requiring PML, twelve layers were used to minimize reflections from the boundary; the 
normalized maximum absorption coefficient, $\sigma$, was 0.25 and increased cubically as fields propagated through the PML layers. The simulations were performed using the commercial software Lumerical FDTD Solutions. ${ }^{9}$

This material is available free of charge via the Internet at http://pubs.acs.org.

\section{References}

(1) Xu, Y. Appl. Opt. 1995, 34, 4573-4588.

(2) Xu, Y. J. Comput. Phys. 1998, 139, 137-165.

(3) Mackowski, D. W. Proceedings: Mathematical and Physical Sciences 1991, 433, year.

(4) Bohren, C. F.; Huffman, D. R. Absorption and Scattering of Light by Small Particles; Wiley, 1998.

(5) Sivanandam, S.; Deepa, S. Introduction to Genetic Algorithms; Springer, 2008.

(6) Pasquale, A. J.; Reinhard, B. M.; Dal Negro, L. ACS Nano 2011, 5, 6578-6585.

(7) Johnson, P. B.; Christy, R. W. Phys. Rev. B 1972, 6, 4370-4379.

(8) Berenger, J.-P. J. Comput. Phys. 1994, 114, 185-200.

(9) Lumerical Solutions, Inc. http: / /www. lumerical.com/.

This material is available free of charge via the Internet at http://pubs . acs . org. 